\title{
Altered CSF Proteomic Profiling of Paediatric Acute Lymphocytic Leukemia Patients with CNS Infiltration
}

\author{
Fei Mo, Xuelei Ma (D), Xiaobei Liu, Ruofan Zhou, Yunuo Zhao, and Hui Zhou \\ Department of Biotherapy, Cancer Center, State Key Laboratory of Biotherapy, West China Hospital, Sichuan University, China \\ Correspondence should be addressed to Xuelei Ma; drmaxuelei@gmail.com
}

Received 5 January 2019; Accepted 9 April 2019; Published 2 May 2019

Academic Editor: Thomas E. Adrian

Copyright (C) 2019 Fei Mo et al. This is an open access article distributed under the Creative Commons Attribution License, which permits unrestricted use, distribution, and reproduction in any medium, provided the original work is properly cited.

\begin{abstract}
Background. For childhood acute lymphocytic leukemia (ALL), central nervous system leukemia (CNSL) is still the main reason of treatment failure. Changes of cerebrospinal fluid (CSF) proteome are deemed to occur after intrathecal chemotherapy. Objective. To find critical CSF biomarkers, which could be utilized to increase diagnostic and prognostic accuracy of CNSL. Methods. We performed proteomic profiling of CSF before and after the treatment of six sporadic paediatric patients diagnosed as ALL with central nervous system (CNS) involvement. CSF samples were properly processed and analyzed through the use of label-free liquid chromatography-tandem mass spectrometry (LC-MS/MS). Results. Among identified 428 unique proteins in all CSF samples, we quantified 10 altered proteins with diverse biological functions after induction chemotherapy. Conclusions. The levels of those 10 proteins change during the treatment of CNSL. Some of the proteins are likely to play a vital biological role as biomarkers for the development of ALL. In addition, our results indicated the feasible and reproducible utility of CSF for diagnosis and prognosis of patients with CNSL.
\end{abstract}

\section{Introduction}

ALL is the most frequent malignancy in children and the peak incidence pertains to 1-5 years age cohort [1]. Recently, a study showed that during 2010-2014 in 14 countries, the lowest rate of 5-year survival of paediatric patients with ALL was of Ecuadorians (49.80\%), and the highest was of Finns (95.20\%), the range of which was elevated $10 \%$ or more compared with that in 1995 [2]. Paediatric ALL has been considered to be potentially curable as a result of the rapidly improved cytogenetic, molecular, and immunophenotyping stratification of leukemic blasts and risk-directed treatment [3-5]. However, CNS relapse still occurs in 3-8\% of the children with ALL and it is a major factor causing death related to cancer of children over 5. Moreover, patients with CNSL are apt to have a relatively poor outcome compared with CNSnegative patients [6]. Therefore, in patients with CNSL, a more sensitive and noninvasive diagnosis method and new biomarkers reflecting treatment response and prognosis are in urgent need [7].

To date, CSF proteomics has rapidly developed and become a new method of diagnosis, treatment, and prognosis of a wide range of diseases, especially nervous system-relative disorders [8], such as Alzheimer, disseminated sclerosis Parkinson, chronic nervous headache, acute brain injury, and mental disorders [9-12]. Increased evidence showed that the proteomic analysis of CSF could also provide candidate protein biomarkers for brain tumours [13], especially glioma [14]. Unfortunately, few researches aimed at finding the CSF proteome change of hematological malignancies [15]. Therefore, we undertook a research focusing on the quantitative proteomics of CSF in patients with nasal-type of extranodal natural killer cell/T-cell lymphoma (NKTCL) [16]. In the present study, we performed a high-throughput quantitative CSF proteomic analysis of patients with CNSL before and after conventional treatment by label-free LC-MS/MS. We found that the levels of ten proteins with different locations and functions significantly changed during the induction chemotherapy for ALL. These proteins are associated with inflammatory processes and tumour development in varying degrees. Thus, this work may provide useful information for molecular mechanisms of ALL development, as well as predicting clinical prognosis of patients with ALL on high risk of CNS infiltration. 


\section{Materials and Methods}

2.1. Participants and CSF Collection. Eligible patients, aged 111 years, had confirmed B-lineage ALL with CNS involvement when the white blood cells were $1-5 / \mu \mathrm{L}$ with detectable leukemic blasts in CSF. These patients received additional intrathecal chemotherapy (ITC) involving methotrexate, cytarabine, and dexamethasone weekly during induction (comprised prednisolone, daunorubicin, vincristine, and pegaspargase according to the protocols of CCCG-ALL2015). CSF samples were obtained by lumbar puncture from subjects at Pediatric Hematology of West China Second University Hospital, Sichuan University. The CSF samples collected before the treatment and after achieving a complete response were divided into two groups: PRE-CSF and POSTCSF, respectively. After standard checks in laboratory, all CSF samples were spun at $3000 \mathrm{rpm}$ under the temperature of $4^{\circ} \mathrm{C}$ for $10 \mathrm{~min}$ to remove any cellular debris and were then stored in aliquots under the temperature of $-80^{\circ} \mathrm{C}$ after $2 \mathrm{~h}$ of harvesting. The Medical Ethics Committee belonging to Sichuan University's West China Hospital confirms the study. Also, the informed consents had been signed by all these patients before they hospitalized.

2.2. CSF Sample Preparation. Aliquots of CSF were dissolved at room temperature. Also, the dilution of a fixed amount of CSF $(30 \mu \mathrm{L})$ was lysed in RIPA buffer $(150 \mathrm{~mm} \mathrm{NaCl}$, $50 \mathrm{~mm}$ Tris- $\mathrm{HCl}$ (pH 7.61), NP-40, 1\% deoxycholic acid) with phosphatase and protease inhibitors for $20 \mathrm{~min}$ on ice. After 10 min's centrifugation at $13000 \mathrm{x}$ g under the temperature of $4^{\circ} \mathrm{C}$, determination of the supernatant protein concentrations was finished by BCA assay. The buffer containing $100 \mathrm{mM}$ $\mathrm{NH}_{4} \mathrm{HCO}_{3}$ was added to obtain the $50 \mathrm{mM} \mathrm{NH}_{4} \mathrm{HCO}_{3}$ 's final protein concentration, making an alkaline environment for the trypsin digestion. Then the samples were reduced with a final concentration of $5 \mathrm{mM}$ DL-Dithiothreitol (DTT) for $1 \mathrm{~h}$ under the temperature of $56^{\circ} \mathrm{C}$. For the purpose of alkylating the cysteines, $55 \mathrm{mM}$ iodoacetamide (IAA) was added to reach the concentration of $15 \mathrm{mM}$, which then reacted in darkness under room temperature for $30 \mathrm{~min}$. Then $30 \mathrm{mM}$ L-cysteine was added to block redundant IAA, and digestion of protein samples was finished with trypsin (Promega) at a ratio of 50:1 (protein to trypsin) under the temperature of $37^{\circ} \mathrm{C}$ overnight under $\mathrm{pH}$ 8.0. Finally, the samples were heated to the temperature of $95^{\circ} \mathrm{C}$ for $10 \mathrm{~min}$ to stop the reaction. Before LC-MS/MS analysis, desalination of the peptides was finished by C18 ZipTip (Millopore, ZTC18S096).

2.3. Liquid Chromatography-Tandem Mass Spectrum Analysis. During the LC-MS/MS analysis process, the same method of our previous report was adopted [16]. In brief, all these lyophilized peptide digests were resuspended in buffer A (2.0\% ACN, 0.1\% FA), after which LC-MS/MS analysis was conducted in triplicate by nanoflow EASY-nLC system, coupled online to Q-Exactive quadrupole-orbitrap mass spectrometer (Thermo Fisher Scientific) with a nanoelectrospray ionization source. The determination of run orders for all the samples was made in a random manner. A $100 \mu \mathrm{m} \times 2 \mathrm{~cm}$ trap column (200A, $5 \mu \mathrm{m}$; Michrom Bioresources) was used to perform separations, and then the trap column was switched with an analytical column of $75 \mu \mathrm{m} \times 12 \mathrm{~cm}(200 \mathrm{~A}, 5 \mu \mathrm{m}$; Michrom Bioresources).

A 3 min column washing was conducted with mobile phase A (2.0\% ACN, 0.1\% FA). The 60-min liquid-chromatography (LC) gradient was used with a rising percentage of buffer B (95.0\% ACN, 0.1\% FA) from initially $4 \%$ to $90 \%$ for peptide elution purposes (flow rate: $300 \mathrm{~nL} / \mathrm{min}$ ). Data were collected by positive ion mode. MS spectra selection scan range was m/z $375-1800$ (resolution: 70,000, m/z: 200). The operation of the LTQ-Orbitrap was finished in datadependent acquisition mode. The value of automatic gain control (AGC) was set at 3e6. The top 15 most intense parent ions (number: 20) were selected and the fragmentation was performed in HCD collision cell (the set mass resolution: 35000). For the purpose of preventing repetitive selection of peptide, a 30 s' dynamic exclusion was applied. Collection of the raw files was acquired using Q-Exactive, after which they were analyzed by Maxquant v1.3. The searching was accomplished according to Swiss-Prot human database. To ensure the confidence in these listed proteins, identification of peptides was made with at least 1 unique peptide with a peptide false discovery rate $<1 \%$ on the basis of Peptide Prophet Algorithm in Elucidator. Five missed trypsin cleavages were allowed; cysteine carbamido methylation was set as a fixed modification in this search.

2.4. Proteomics Enrichment Analysis. After filtering the data set, enrichment analysis of the CSF proteome was taken in Gene Ontology (GO). Then, the identified proteins' Kyoto Encyclopedia of Genes and Genomes (KEGG) pathways were carried out using David 6.8 (https://david-d.ncifcrf.gov/). Significantly altered-protein expression profiles were present by heatmap in Mev software. Finally, we took visualized known STRING actions (https://string-db.org/) for significantly changed CSF proteins [17].

2.5. Western Blot. CSF samples were diluted in 5x SDSPAGE sample loading buffer (Beyotime) after determining the protein concentration and heated at $95^{\circ} \mathrm{C}$ for 10 minutes. Fifty micrograms of total proteins from each sample were loaded into each well, then separated on $12.5 \%$ SDS-PAGE gel (Bio-Rad) and transferred to the polyvinylidene difluoride membrane (Millipore). After incubation with blocking solution (containing 5\% milk) for $120 \mathrm{~min}$ at room temperature, membranes were incubated with the following primary antibodies overnight at $4^{\circ} \mathrm{C}$ : rabbit-anti-HRG (Abcam), rabbitanti-SPARC (Abcam). Then, membranes were washed three times and incubated with horseradish peroxidase-conjugated secondary antibody (Thermo Fisher Scientific) at $37^{\circ} \mathrm{C}$ for $1 \mathrm{~h}$. Chemiluminescent visualization (Clinx Science Instruments) was used to visualize the signals. Western blot data were normalized by Image J software.

2.6. Statistical Analysis. Multiple comparisons of PRE-CSF and POST-CSF samples were performed using paired $\mathrm{t}$ test. The analysis of protein profiles was implemented for candidate proteins using univariate analysis. We filtered our list of significant altered proteins at $\mathrm{p}$ value $<0.05$. $\mathrm{P}$ values 


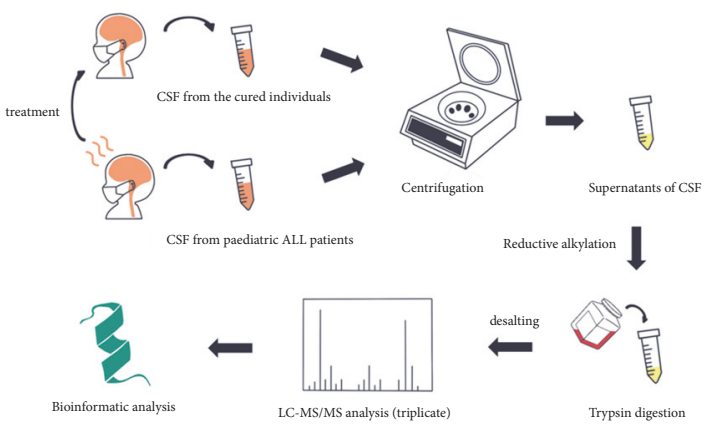

(a)

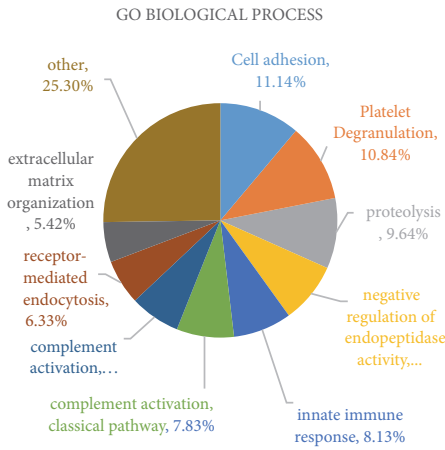

(c)

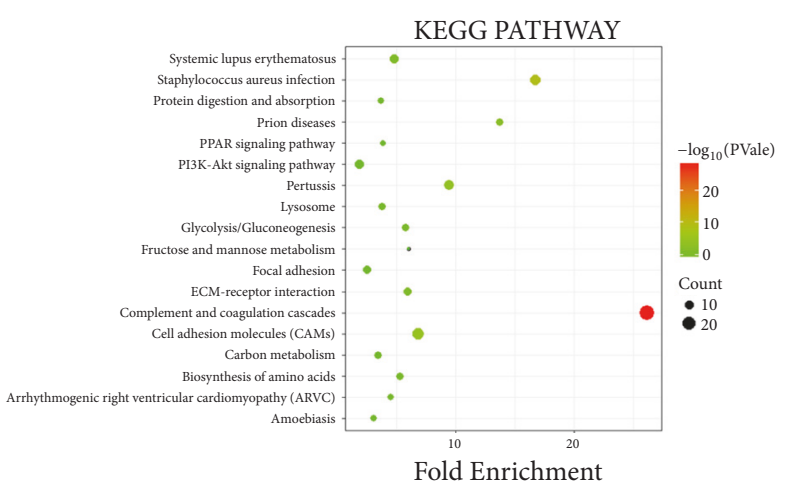

(b)

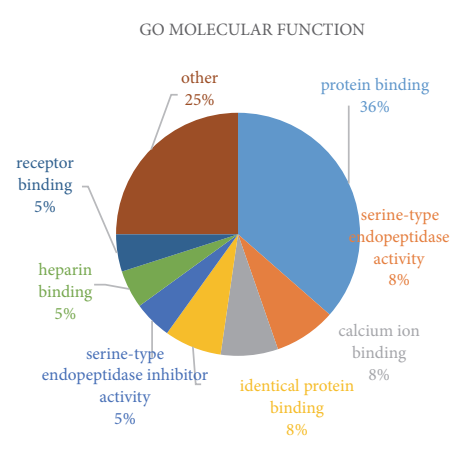

(d)

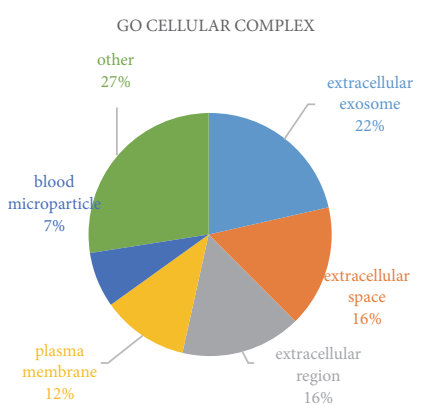

(e)

FIGURE 1: The comprehensive proteomics analysis of CSF with CNSL. (a) The experimental work flow. The process of sample preparation, data acquisition, and analysis is shown in the diagram. ALL, leukeamia. (b) In-depth KEGG-pathway analysis of all identified proteins in PRE-CSF samples. (c), (d), and (e) Gene Ontology (GO) analysis of all identified proteins expressed in PRE-CSF samples. All identified proteins were put into the three GO domains (BP, MF, and CC). The names and percentages of term are located next to their position on the charts.

were calculated in accordance with Mann-Whitney U test for nonparametric data, or the two-tailed t-test for parametric data. The data were shown as means \pm standard deviations (SD).

\section{Results}

3.1. Global CSF Protein Analysis. Ultimately, six eligible patients were included in our study. The demonstration of our experimental workflow was presented in Figure 1(a). To investigate the proteomic profile of CSF in patients with CNSL, we collected PRE- and POST-CSF samples and used the same volume of samples for subsequent laboratory tests and analysis. Within these pooled CSF samples, an average of 428 unique proteins of all PRE-CSF and POSTCSF samples was identified by measuring peptides signal intensity for protein abundance (Supplementary Table S1). During the treatment, the following pathway was revealed activated by depth KEGG-pathway analysis: complement and coagulation cascades, related to the usage of antitumor drugs during induction therapy to ALL [18] (Figure 1(b)). Next, we grouped those proteins according to annotations in Gene Ontology (GO) domains, including 'Biological Process (BP)', 'Molecular Function (MF)', and 'Cellular Complex (CC)', to determine the constitutions of the CSF proteome. Then, the percentage representation of each GO subcategory domain was demonstrated with all expressed proteins. There was no substantial difference among the three subcategories proportions between pretreated and posttreated groups of patients with ALL (Supplementary Figure S1). The analysis in BP ontology showed significant protein enrichment in the cell adhesion, platelet degranulation, and proteolysis (Figure 1(c)). MF analysis indicated that most of the identified proteins were noted as protein binding and calcium ion binding as well as serine-type endopeptidase inhibitor activity (Figure 1(d)). Analysis of CC ontology mainly pointed towards extracellular exosome, extracellular space, and extracellular region (Figure 1(e)).

3.2. Label-Free Quantification of Relevant Protein Abundance. Aiming at identifying differentially expressed proteins in these PRE-CSF and POST-CSF samples, we performed a strict $\mathrm{t}$-test difference criteria (Method section) and several statistically significant proteins were identified (Table 1). The expression of all these proteins was presented in Figure 2(a). Our label-free quantification demonstrated that the following seven proteins were upregulated: histidine rich glycoprotein (HRG), kallikrein related peptidase 6 (KLK6), carnosine dipeptidase 1 (CNDP1), alpha-2-macroglobulin (A2M), complement factor $\mathrm{H}(\mathrm{CFH})$, complement $\mathrm{C} 4 \mathrm{~A}$ (C4A), and apolipoprotein A1 (APOA1). On the contrary, WAP, follistatin/kazal, immunoglobulin, kunitz and netrin 
TABLE 1: Statistically significant increased or decreased proteins in ALL samples during induction with $\mathrm{P}<0.05$.

\begin{tabular}{|c|c|c|c|c|}
\hline Protein & Gene name & Protein ID & Change trend & $P$ value \\
\hline complement C4A (Rodgers blood group) & C4A & P0C0L4 & $\uparrow$ & 0.011 \\
\hline Histidine rich glycoprotein & HRG & P04196 & $\uparrow$ & 0.016 \\
\hline apolipoprotein A1 & APOA1 & P02647 & $\uparrow$ & 0.025 \\
\hline kallikrein related peptidase 6 & KLK6 & Q92876 & $\uparrow$ & 0.025 \\
\hline carnosine dipeptidase 1 & CNDP1 & Q96KN2 & $\uparrow$ & 0.026 \\
\hline complement factor $\mathrm{H}$ & $\mathrm{CFH}$ & P08603 & $\uparrow$ & 0.029 \\
\hline alpha-2-macroglobulin & $\mathrm{A} 2 \mathrm{M}$ & P01023 & $\uparrow$ & 0.05 \\
\hline apolipoprotein D & APOD & P05090 & $\downarrow$ & 0.018 \\
\hline $\begin{array}{l}\text { WAP, follistatin/kazal, immunoglobulin, } \\
\text { kunitz and netrin domain containing } 2\end{array}$ & WFIKKN2 & Q8TEU8 & $\downarrow$ & 0.024 \\
\hline secreted protein acidic and cysteine rich & SPARC & P09486 & $\downarrow$ & 0.037 \\
\hline
\end{tabular}
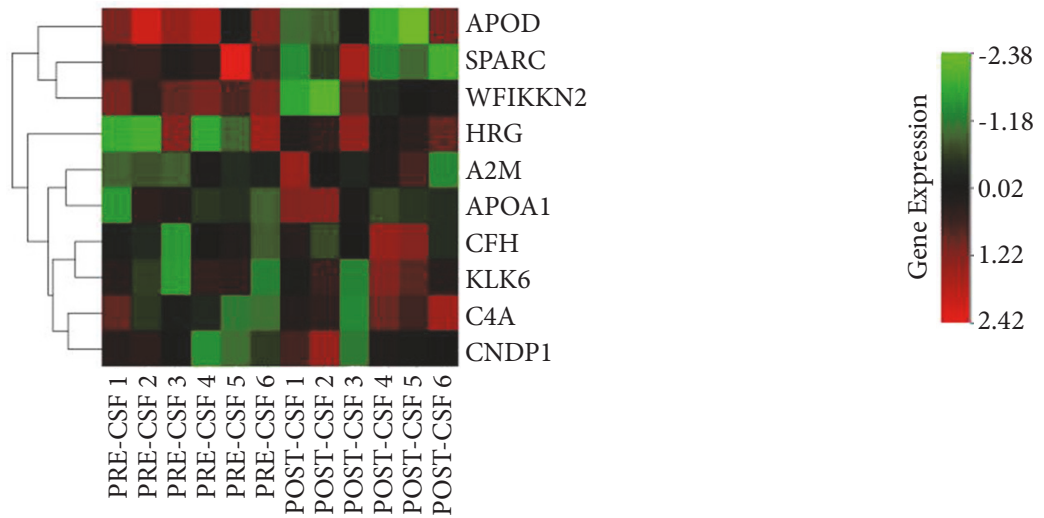

(a)

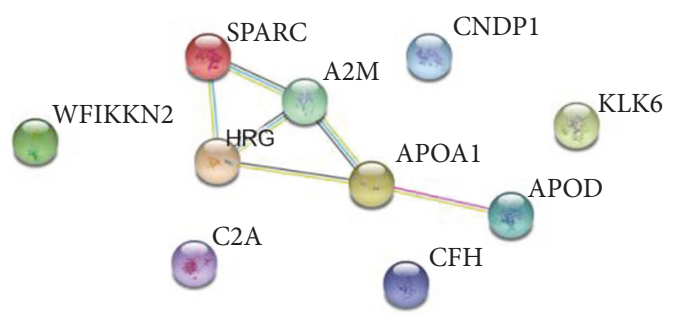

(b)

FIGURE 2: Quantification of changed CSF proteins during the treatment of CNSL. (a) Heatmap analysis of deproteins in PRE-CSF and POSTCSF group: unsupervised clustering analysis showing expression profiles of DE proteins with Euclidean Distance of proteins. (b) The alteredprotein interactions generated by STRING analysis. Required confidence (score): medium confidence (0.400). Edge width reflects the strength of the STRING action score and the edge length is arbitrary.

domain including 2 (WFIKKN2), secreted protein acidic as well as cysteine rich (SPARC), apolipoprotein D (APOD) were significantly downregulated. In addition, we used western blotting technique to validate the LC-MS/MS results, demonstrated in Supplementary Figure S2. HRG and SPARC were selected for the confirmation of the low intensity and potential applied value as the biomarkers of ALL. Consistent with our LC-MS/MS results, the content of SPARC was obviously decreased $(p=0.044)$ and HRG had the trend of increasing $(\mathrm{p}=0.072)$ after treatment of ALL. The protein interaction among these 10 proteins is showed in Figure 2(b). Therefore, these proteins are possibly related to ALL development directly or indirectly, and their changes during the treatment might be used as an indication of the better therapeutic effects in CNS.

\section{Discussion}

In this research, the label-free LC-MS/MS was adopted for constructing overall CSF proteomic profiles of pediatric 
CNSL samples. Strong concordance could be seen in the proportion of identified proteins assigned to $\mathrm{BP}, \mathrm{MF}$, and CC of GO domains between PRE and POST-CSF samples from our data, demonstrating that the overall composition of CSF proteome is relatively stable [16]. In addition, the different points in relative levels of CSF proteins were detected in CNSL patients during induction. As subclinical CNS manifestation is in most patients, CNS-directed therapy is critical for increasing leukemia-free survival rates, even in nondetectable CNSL pediatric patients [19]. Intrathecal chemotherapy (ITC) with methotrexate or cytarabine is commonly used to prevent or treat CNSL. However, various adverse events are likely to occur, including severe gastrointestinal, hepatic and neurological toxicities, leukoencephalopathy, and polyradiculopathy $[20,21]$. Therefore, improving therapy efficacy and reducing toxicity are the current focus of ALL management [22]. CSF is proximate to CNS tissue microenvironment and has relative simplex protein contents; thus, it is preferentially considered as an ideal biomarker source for neurological disorders [23]. By analyzing the changes of expressed proteins during ITC in patients with CNSL, we could find new markers to develop alternative CNSL-related diagnosis, prognosis, and treatment strategies.

In this work, we found the levels of three proteins (SPARC, WFIKKN2, and APOD) decreased during CNSL treatment. SPARC is a $32 \mathrm{kDa}$ secreted glycoprotein that regulates interaction of cell-extracellular matrix proteins to promote cell adhesion and migration [24]. It is also reported to be important for tissue remodelling, angiogenesis, and tumourigenesis through activation of growth factors and matrix metalloproteinases [25-28]. Increased expression of SPARC has been found to play a protumorigenic and prometastatic role to many malignant tumours, such as brain, lung, breast, pancreas, skin, and kidney [29]. Another study showed that the downregulated or absent expression of SPARC is related to high-speed progression of human colon cancer [30]. Thus, SPARC may have complex function in the development of human solid tumours. However, there are few studies discussing the role of SPARC in the formation of hematological malignances. Recently, accumulating evidence suggested that SPARC overexpression is associated with poor outcome in acute myeloid leukemia (AML) patients and promotes the growth of aggressive leukemia cell in murine models. In addition, knockdown of SPARC could inhibit AML cell proliferation through inducing cell cycle arrest at Phase G1/G0 and inhibit active p53-induced apoptosis through raising the levels of $\mathrm{p} 53$, caspase- 9 , caspase-3, and Fas $[24,31]$. As a marker of poor prognosis and a potential therapeutic target of AML, a similar mechanism may be helpful for inhibiting ALL development, and the decreased level of SPARC may account for a better prognosis of CNSL, since the levels of SPARC significantly declined in subjects when they CR was achieved in our study. WFIKKN2 is a large extracellular multidomain protein, containing a WAP, an immunoglobulin, a follistatin domain (binds mature growth factor), an NTR domain, and two Kunitz-type protease inhibitor domains [32]. It is known to positively activate PI3K pathway, which could regulate cancer cell growth and progression [33]. Moreover, it can promote the combination of TGF $\beta$ family members to the receptor to regulate the balanced relation between the activation of non-Smad and Smad pathways of TGF $\beta$ [34]. As a 33-kDa glycoprotein component of high-density lipoprotein in plasma, Apo D is observably expressed by glial cells in brain [35]. It is essential for the development and repair of nervous system as an important regulator of lipid trafficking and conferring protection from cell oxidative stress $[36,37]$. Apo D could also act as a nonspecific stress protein leading to cell growth arrest, and it is upregulated under several pathological situations such as cancers and neurodegenerative diseases $[38,39]$.

Increased levels of C4A and CFH were involved in complement and coagulation cascades pathway [40]. Furthermore, $\mathrm{CFH}$ inhibits endothelial cell migration and angiogenesis by indirectly regulating angiogenic effectors of complement components, including $\mathrm{C} 3 \mathrm{a}$ and $\mathrm{C} 5 \mathrm{a}$, leading to lower vascular endothelial growth factor (VEGF) expression and transformation of growth factor- $\beta$ (TGF- $\beta$ ) [41-43]. The upregulated expression of $\mathrm{CFH}$ might be beneficial to confront VEGF produced by ALL cells, as VEGF has been proved to promote leukemia cell infiltration into CNS [36]. As a $720-\mathrm{kDa}$ glycoprotein, A2M is mainly produced by liver Kupffer cells. A2M is also involved in inflammatory reaction as a proteinase inhibitor and carrier for interleukin-6 (IL6) [37]. It has been shown to decrease in some pathological conditions such as hematologic malignancy and advanced prostate cancer $[44,45]$. It was demonstrated previously that the interaction of it with the lipoprotein receptorrelated protein-1(LRP1) of low-density led to an inhibition of tumour cell proliferation, migration, and invasion in astrocytoma malignancy [46]. HRG is an $75 \mathrm{kDa}$ a2glycoprotein produced by liver, which keeps high levels in plasma and acts as an acute phase reactant [47]. HRG is involved in numerous biologic processes, such as cell adhesion and proliferation, angiogenesis as well as coagulation and platelet activation [48]. It is also reported that HRG inhibits tumour growth and vascularization, remodels transition from epithelial to mesenchymal, and regulates the formation of protumorigenic microenvironment by skewing tumour-associated macrophages polarization from the M2like towards M1-like phenotype [49-51]. Besides, HRG promotes antitumor immune responses and vessel normalization to enhance chemotherapy. KLK6, a member of the secreted serine protease family of protein-cleaving enzymes, is also defined as an epigenetically regulated tumour suppressor [52]. KLK6 expression resulted in significant downregulation of vimentin, a critical marker of epithelial-to-mesenchymal transition of tumour cells [53]. APOA1, the major protein component of high-density lipoproteins (HDL) particles in plasma, suppressed neutrophil recruitment in inflammation to avoid excessive inflammatory response [54]. Furthermore, it is a potential antiangiogenic factor to suppress tumour progression by targeting c-Src/ERK signaling pathways [55, 56]. Carnosinase 1 (CNDP1), primarily synthesized in brain, secreted into CSF, and finally secreted into the blood, is likely to be important in the maintenance and bioavailability of carnosine, a drug for tumours. It could be verified that CNDP1 has antiproliferative properties on different malignant cells for its therapeutic potential against tumours 
including glioblastoma and metastatic prostate cancer. Its deficiency has been related to many kinds of neurologic deficits and cancer cachexia [57-59]. Several statistically altered proteins have been previously proved to play a role of regulation in malignant cells or microenvironment of cancers, which might be related to the molecular mechanisms of ALL development and prognosis of patients with CNSL.

Collectively, we constructed CSF's label-free LC-MS/MS proteomic profiles in patients suffering from CNSL, which helps us identify valuable biomarkers to understand the development of ALL with CNS infiltration. It is noteworthy that those altered proteins should be furtherly investigated as predictive markers of ALL patients with CNS infiltration, some of which may have a prospect of becoming the new therapeutic targets in CNSL. However, this study has some limitations. For example, the size of samples is small since only 6 subjects are covered, and the period of observation is relatively short. Therefore, we will enlarge the sample size and prolong the observation time in our next study, hoping to further verify the roles of those altered proteins in paediatric patients of ALL with CNS infiltration.

\section{Abbreviations}

\begin{tabular}{|c|c|}
\hline A2M: & Alpha-2-macroglobulin \\
\hline ACN: & Acetonitrile \\
\hline AGC: & Automatic gain control \\
\hline ALL: & Acute lymphocytic leuk(a)emia \\
\hline AML: & Acute myeloid leuk(a)emia \\
\hline APOA1: & Apolipoprotein A1 \\
\hline APOD: & Apolipoprotein D \\
\hline BP: & Biological Process \\
\hline C4A: & Complement C4A \\
\hline CC: & Cellular Complex \\
\hline CFH: & Complement factor $\mathrm{H}$ \\
\hline CID: & Collision induced dissociation \\
\hline CNDP1: & Carnosine dipeptidase 1 \\
\hline CNS: & Central nervous system \\
\hline CNSL: & Central nervous system leuk(a)emia \\
\hline CSF: & Cerebrospinal fluid \\
\hline DTT: & DL-Dithiothreitol \\
\hline FA: & Formic acid \\
\hline GO: & Gene Ontology \\
\hline HCD: & $\begin{array}{l}\text { Higher energy Collision induced } \\
\text { Dissociation }\end{array}$ \\
\hline HDL: & High-density lipoproteins \\
\hline HRG: & Histidine rich glycoprotein \\
\hline IAA: & Iodoacetamide \\
\hline IL-6: & Interleukin-6 \\
\hline IT: & Intrathecal therapy \\
\hline ITC: & Intrathecal chemotherapy \\
\hline KEGG: & $\begin{array}{l}\text { Kyoto Encyclopedia of Genes and } \\
\text { Genomes }\end{array}$ \\
\hline KLK6: & Kallikrein related peptidase 6 \\
\hline LC: & Liquid-chromatography \\
\hline LC-MS/MS: & $\begin{array}{l}\text { Liquid chromatography-tandem mass } \\
\text { spectrometry }\end{array}$ \\
\hline LRP1: & Lipoprotein receptor-related protein-1 \\
\hline LTQ: & Linear trap \\
\hline
\end{tabular}

MF: $\quad$ Molecular Function

NKTCL: Natural killer cell/T-cell lymphoma

POST-CSF: After achieving a complete response

PRE-CSF: Before the treatment

SD: $\quad$ Standard deviations

SPARC: $\quad$ Secreted protein acidic and cysteine rich

TGF- $\beta$ : $\quad$ Transforming growth factor- $\beta$

VEGF: Vascular endothelial growth factor

WAP: Whey acidic protein

WFIKKN2: WAP, follistatin/kazal, immunoglobulin, kunitz and netrin domain containing 2.

\section{Data Availability}

The data from LC-MS/MS analysis used to support the findings of this study are included within the article.

\section{Conflicts of Interest}

The authors declare that there are no conflicts of interest.

\section{Authors' Contributions}

Fei Mo and Xuelei Ma contributed equally to this work.

\section{Acknowledgments}

We appreciate the patients and their families for participating in the study.

\section{Supplementary Materials}

Supplementary Figure S1: Gene Ontology (GO) analysis of all identified proteins expressed in POST-CSF samples. Supplementary Figure S2: validation of SPARC and HRG protein peaks by western blot. (A) Immunoblot of SPARC and HRG proteins in PRE-CSF and POST-CSF samples. Fifty micrograms of PRE-CSF (lanes 1-3) or POST-CSF (lanes 4-6) CSF were subjected to the lanes. (B) and (C) The relative quantification of SPARC and HRG proteins from panels (A) using Image J software, respectively. Supplementary Table S1: an average of 428 unique proteins of all PRE-CSF and POSTCSF samples was identified by measuring peptides signal intensity for protein abundance. (Supplementary Materials)

\section{References}

[1] S. Chiaretti, A. Vitale, G. Cazzaniga et al., "Clinico-biological features of 5202 patients with acute lymphoblastic leukemia enrolled in the Italian AIEOP and GIMEMA protocols and stratified in age cohorts," Haematologica, vol. 98, no. 11, pp. 1702-1710, 2013.

[2] C. Allemani, T. Matsuda, C. V. Di et al., "Global surveillance of trends in cancer survival 2000-14 (CONCORD-3): analysis of individual records for 37513025 patients diagnosed with one of 18 cancers from 322 population-based registries in 71 countries," Lancet, vol. 391, article no. 10125, pp. 1023-1075, 2018. 
[3] S. P. Hunger and C. G. Mullighan, "Acute lymphoblastic leukemia in children," The New England Journal of Medicine, vol. 373, no. 16, pp. 1541-1552, 2015.

[4] A. T. M. A. Rahman, S. K. Gupta, M. A. Mannan, and K. Nahar, "Augmented post-induction therapy for children with high-risk acute lymphoblastic leukemia and a slow response to initial therapy," Kathmandu University Medical Journal, vol. 10, no. 40, pp. 53-59, 2012.

[5] C.-H. Pui, "Acute lymphoblastic leukemia in children," Current Opinion in Oncology, vol. 12, no. 1, pp. 3-12, 2000.

[6] R. K. Marwaha, K. P. Kulkarni, D. Bansal, and A. Trehan, "Central nervous system involvement at presentation in childhood acute lymphoblastic leukemia: Management experience and lessons," Leukemia \& Lymphoma, vol. 51, no. 2, pp. 261-268, 2010.

[7] C. Pui and S. C. Howard, "Current management and challenges of malignant disease in the CNS in paediatric leukaemia," The Lancet Oncology, vol. 9, no. 3, pp. 257-268, 2008.

[8] A. K. Anagnostopoulos and G. T. Tsangaris, "The proteomics of pediatric brain tumors," Expert Review of Proteomics, vol. 11, no. 5, pp. 641-648, 2014.

[9] P. Davidsson, L. Paulson, C. Hesse, K. Blennow, and C. L. Nilsson, "Proteome studies of human cerebrospinal fluid and brain tissue using a preparative two-dimensional electophoresis approach prior to mass spectrometry," Proteomics, vol. 1, no. 3, pp. 444-452, 2001.

[10] C. Rohlff, "Proteomics in molecular medicine: Applications in central nervous systems disorders," Electrophoresis, vol. 21, no. 6, pp. 1227-1234, 2000.

[11] P.-P. Zheng, T. M. Luider, R. Pieters et al., "Identification of tumor-related proteins by proteomic analysis of cerebrospinal fluid from patients with primary brain tumors," Journal of Neuropathology \& Experimental Neurology, vol. 62, no. 8, pp. 855-862, 2003.

[12] C. Gineste, L. Ho, P. Pompl, M. Bianchi, and G. M. Pasinetti, "High-throughput proteomics and protein biomarker discovery in an experimental model of inflammatory hyperalgesia: effects of nimesulide," Drugs, vol. 63, Supplement 1, pp. 23-29, 2003.

[13] N. Samuel, M. Remke, J. T. Rutka, B. Raught, and D. Malkin, "Proteomic analyses of CSF aimed at biomarker development for pediatric brain tumors," Journal of Neuro-Oncology, vol. 118, no. 2, pp. 225-238, 2014.

[14] F. Shen, Y. Zhang, Y. Yao et al., "Proteomic analysis of cerebrospinal fluid: Toward the identification of biomarkers for gliomas," Neurosurgical Review, vol. 37, no. 3, pp. 367-380, 2014.

[15] S. Roy, S. A. Josephson, J. Fridlyand et al., "Protein biomarker identification in the CSF of patients with CNS lymphoma," Journal of Clinical Oncology, vol. 26, no. 1, pp. 96-105, 2008.

[16] Y. Gong, W. Pu, H. Jin et al., "Quantitative proteomics of CSF reveals potential predicted biomarkers for extranodal NK-/Tcell lymphoma of nasal-type with ethmoidal sinus metastasis," Life Sciences, vol. 198, pp. 94-98, 2018.

[17] C. von Mering, M. Huynen, D. Jaeggi, S. Schmidt, P. Bork, and B. Snel, "STRING: a database of predicted functional associations between proteins," Nucleic Acids Research, vol. 31, no. 1, pp. 258261, 2003.

[18] X. Dong, J. Shi, J. Zhou et al., "Chemotherapy induces enhanced procoagulant activity through phosphatidylserine exposure in acute lymphoblastic leukemia," Thrombosis Research, vol. 132, no. 5, pp. 614-620, 2013.
[19] I. Schuitema, S. Deprez, W. Van Hecke et al., "Accelerated aging, decreased white matter integrity, and associated neuropsychological dysfunction 25 years after pediatric lymphoid malignancies," Journal of Clinical Oncology, vol. 31, no. 27, pp. 3378-3388, 2013.

[20] M. Suematsu, T. Imamura, T. Chiyonobu, S. Osone, and H. Hosoi, "Lumbosacral polyradiculopathy after intrathecal chemotherapy in pediatric acute lymphoblastic leukemia," International Journal of Hematology, vol. 107, no. 5, pp. 499-501, 2018.

[21] R. Franca, P. Rebora, N. Bertorello et al., "Pharmacogenetics and induction/consolidation therapy toxicities in acute lymphoblastic leukemia patients treated with AIEOP-BFM ALL 2000 protocol," The Pharmacogenomics Journal, vol. 17, no. 1, pp. 4-10, 2017.

[22] S. Pillozzi, B. Accordi, P. Rebora et al., "Differential expression of hERG1A and hERG1B genes in pediatric acute lymphoblastic leukemia identifies different prognostic subgroups," Leukemia, vol. 28, no. 6, pp. 1352-1355, 2014.

[23] M. A. Collins, J. An, B. L. Hood, T. P. Conrads, and R. P. Bowser, "Label-free LC-MS/MS proteomic analysis of cerebrospinal fluid identifies protein/pathway alterations and candidate biomarkers for amyotrophic lateral sclerosis," Journal of Proteome Research, vol. 14, no. 11, pp. 4486-4501, 2015.

[24] H. Alachkar, R. Santhanam, K. Maharry et al., "SPARC promotes leukemic cell growth and predicts acute myeloid leukemia outcome," The Journal of Clinical Investigation, vol. 124, no. 4, pp. 1512-1524, 2014.

[25] M. Tichet, V. Prod'Homme, N. Fenouille et al., "Tumourderived SPARC drives vascular permeability and extravasation through endothelial VCAM1 signalling to promote metastasis," Nature Communications, vol. 6, no. 1, article no. 6993, 2015.

[26] T. F. Lane and E. H. Sage, "The biology of SPARC, a protein that modulates cell-matrix interactions," The FASEB Journal, vol. 8, no. 2, pp. 163-173, 1994.

[27] Y. Kato, J.-M. Lewalle, Y. Baba et al., "Induction of SPARC by VEGF in human vascular endothelial cells," Biochemical and Biophysical Research Communications, vol. 287, no. 2, pp. 422426, 2001.

[28] B. J. Schiemann, J. R. Neil, and W. P. Schiemann, "SPARC inhibits epithelial cell proliferation in part through stimulation of the transforming growth factor- $\beta$-signaling system," Molecular Biology of the Cell (MBoC), vol. 14, no. 10, pp. 3977-3988, 2003.

[29] O. L. Podhajcer, L. G. Benedetti, M. R. Girotti, F. Prada, E. Salvatierra, and A. S. Llera, "The role of the matricellular protein SPARC in the dynamic interaction between the tumor and the host," Cancer and Metastasis Reviews, vol. 27, no. 4, pp. 691-705, 2008.

[30] E. Yang, J. K. Hyun, H. K. Kwi, H. Rhee, K. K. Nam, and H. Kim, "Frequent inactivation of SPARC by promoter hypermethylation in colon cancers," International Journal of Cancer, vol. 121, no. 3, pp. 567-575, 2007.

[31] Q. Nian, Q. Xiao, L. Wang et al., "SPARC silencing inhibits the growth of acute myeloid leukemia transformed from myelodysplastic syndrome via induction of cell cycle arrest and apoptosis," International Journal of Molecular Medicine, vol. 33, no. 4, pp. 856-862, 2014.

[32] K. Kondás, G. Szláma, M. Trexler, and L. Patthy, "Both WFIKKN1 and WFIKKN2 have high affinity for growth and differentiation factors 8 and 11," The Journal of Biological Chemistry, vol. 283, no. 35, pp. 23677-23684, 2008. 
[33] J. Daragmeh, W. Barriah, B. Saad, and H. Zaid, "Analysis of PI3K pathway components in human cancers," Oncology Letters, vol. 11, no. 4, pp. 2913-2918, 2016.

[34] O. Monestier and V. Blanquet, "WFIKKN1 and WFIKKN2: "Companion" proteins regulating TGFB activity," Cytokine \& Growth Factor Reviews, vol. 32, pp. 75-84, 2016.

[35] E. Rassart, A. Bedirian, S. Do Carmo et al., "Apolipoprotein D," Biochimica et Biophysica Acta (BBA) - Protein Structure and Molecular Enzymology, vol. 1482, no. 1-2, pp. 185-198, 2000.

[36] V. Münch, L. Trentin, J. Herzig et al., "Central nervous system involvement in acute lymphoblastic leukemia is mediated by vascular endothelial growth factor," Blood, vol. 130, no. 5, pp. 643-654, 2017.

[37] S. Lyoumi, F. Tamion, J. Petit et al., "Induction and modulation of acute-phase response by protein malnutrition in rats: Comparative effect of systemic and localized inflammation on interleukin-6 and acute-phase protein synthesis," Journal of Nutrition, vol. 128, no. 2, pp. 166-174, 1998.

[38] L.-C. Levros, S. D. Carmo, E. Edouard, P. Legault, C. Charfi, and E. Rassart, "Characterization of nuclear factors modulating the apolipoprotein D promoter during growth arrest: Implication of PARP-1, APEX-1 and ERK1/2 catalytic activities," Biochimica et Biophysica Acta (BBA) - Molecular Cell Research, vol. 1803, no. 9, pp. 1062-1071, 2010.

[39] S. Do Carmo, L. Levros, and E. Rassart, "Modulation of apolipoprotein D expression and translocation under specific stress conditions," Biochimica et Biophysica Acta (BBA) - Molecular Cell Research, vol. 1773, no. 6, pp. 954-969, 2007.

[40] K. Ikeda, K. Nagasawa, T. Horiuchi, T. Tsuru, H. Nishizaka, and Y. Niho, "C5a induces tissue factor activity on endothelial cells," Thrombosis and Haemostasis, vol. 77, no. 2, pp. 394-398, 1997.

[41] N. S. Bora, S. Kaliappan, P. Jha et al., "Complement activation via alternative pathway is critical in the development of laserinduced choroidal neovascularization: role of factor $\mathrm{B}$ and factor H," The Journal of Immunology, vol. 177, no. 3, pp. 18721878, 2006.

[42] M. Nozaki, B. J. Raisler, E. Sakurai et al., "Drusen complement components C3a and C5a promote choroidal neovascularization," Proceedings of the National Acadamy of Sciences of the United States of America, vol. 103, no. 7, pp. 2328-2333, 2006.

[43] J. Liu and J. Hoh, "Loss of complement factor $\mathrm{H}$ in plasma increases endothelial cell migration," Journal of Cancer, vol. 8, no. 12, pp. 2184-2190, 2017.

[44] Y. Kanoh, H. Ohtani, S. Egawa, S. Baba, and T. Akahoshi, "Clinicopathological characteristics of androgen-dependent advanced prostate cancer patients with $\alpha$ 2-macroglobulin deficiency," International Journal of Oncology, vol. 41, no. 1, pp. 39-45, 2012.

[45] Y. Kanoh, H. Ohtani, and K. Koshiba, "Studies on alpha 2 macroglobulin deficiency in association with cancer metastasis," Japanese Journal of Clinical Immunology, vol. 20, no. 1, pp. 30-43, 1997.

[46] I. Lindner, N. Y. Hemdan, M. Buchold et al., "Alpha2-macroglobulin inhibits the malignant properties of astrocytoma cells by impeding beta-catenin signaling," Cancer Research, vol. 70, no. 1, pp. 277-287, 2010.

[47] K. Saigo, A. Yoshida, R. Ryo, N. Yamaguchi, and L. L. K. Leung, "Histidine-rich glycoprotein as a negative acute phase reactant," American Journal of Hematology, vol. 34, no. 2, pp. 149-150, 1990.

[48] I. K. H. Poon, K. K. Patel, D. S. Davis, C. R. Parish, and M. D. Hulett, "Histidine-rich glycoprotein: The Swiss Army knife of mammalian plasma," Blood, vol. 117, no. 7, pp. 2093-2101, 2011.
[49] M. Matboli, S. Eissa, and H. Said, "Evaluation of histidine-rich glycoprotein tissue RNA and serum protein as novel markers for breast cancer," Medical Oncology, vol. 31, no. 4, article no. 897, 2014.

[50] J. Cedervall, Y. Zhang, M. Ringvall et al., "HRG regulates tumor progression, epithelial to mesenchymal transition and metastasis via platelet-induced signaling in the pre-tumorigenic microenvironment," Angiogenesis, vol. 16, no. 4, pp. 889-902, 2013.

[51] C. Rolny, M. Mazzone, S. Tugues et al., "HRG inhibits tumor growth and metastasis by inducing macrophage polarization and vessel normalization through downregulation of PlGF," Cancer Cell, vol. 19, no. 1, pp. 31-44, 2011.

[52] A. Anisowicz, G. Sotiropoulou, G. Stenman, S. C. Mok, and R. Sager, "A novel protease homolog differentially expressed in breast and ovarian cancer," Molecular Medicine, vol. 2, no. 5, pp. 624-636, 1996.

[53] G. Pampalakis, E. Prosnikli, T. Agalioti, A. Vlahou, V. Zoumpourlis, and G. Sotiropoulou, "A tumor-protective role for Human kallikrein-related peptidase 6 in breast cancer mediated by inhibition of epithelial-to-mesenchymal transition," Cancer Research, vol. 69, no. 9, pp. 3779-3787, 2009.

[54] M. Huang, Y. Gong, J. Grondolsky, and J. Hoover-Plow, "Lp(a)/ apo(a) modulate MMP-9 activation and neutrophil cytokines in vivo in inflammation to regulate leukocyte recruitment," The American Journal of Pathology, vol. 184, no. 5, pp. 1503-1517, 2014.

[55] J.-S. Kim, J.-H. Chang, H.-K. Yu et al., "Inhibition of angiogenesis and angiogenesis-dependent tumor growth by the cryptic kringle fragments of human apolipoprotein(a)," The Journal of Biological Chemistry, vol. 278, no. 31, pp. 29000-29008, 2003.

[56] Z. Yi, S. Cho, H. Zhao et al., "A novel peptide from human apolipoprotein(a) inhibits angiogenesis and tumor growth by targeting c-Src phosphorylation in VEGF-induced human umbilical endothelial cells," International Journal of Cancer, vol. 124, no. 4, pp. 843-852, 2009.

[57] P. Schoen, H. Everts, T. De Boer, and W. Van Oeveren, "Serum carnosinase activity in plasma and serum: validation of a method and values in cardiopulmonary bypass surgery," Clinical Chemistry, vol. 49, no. 11, pp. 1930-1932, 2003.

[58] J. F. Lenney, S. C. Peppers, C. M. Kucera, and O. Sjaastad, "Homocamosinosis: lack of serum carnosinase is the defect probably responsible for elevated brain and CSF homocarnosine," Clinica Chimica Acta, vol. 132, no. 2, pp. 157-165, 1983.

[59] W. S. Wassif, R. A. Sherwood, A. Amir et al., "Serum carnosinase activities in central nervous system disorders," Clinica Chimica Acta, vol. 225, no. 1, pp. 57-64, 1994. 


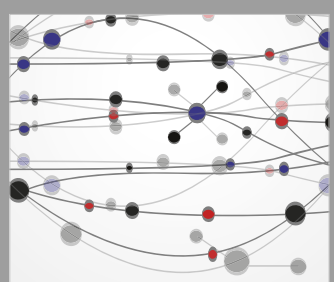

The Scientific World Journal
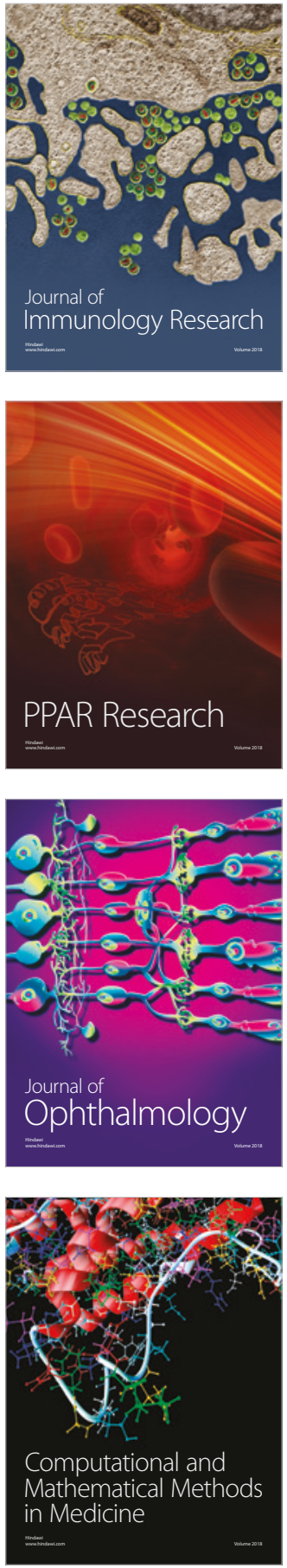

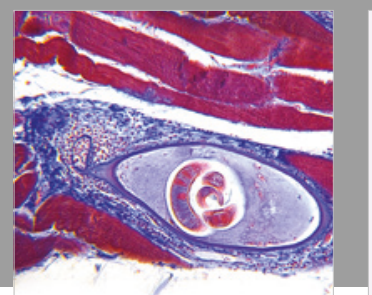

Gastroenterology Research and Practice

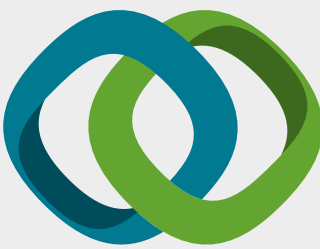

\section{Hindawi}

Submit your manuscripts at

www.hindawi.com
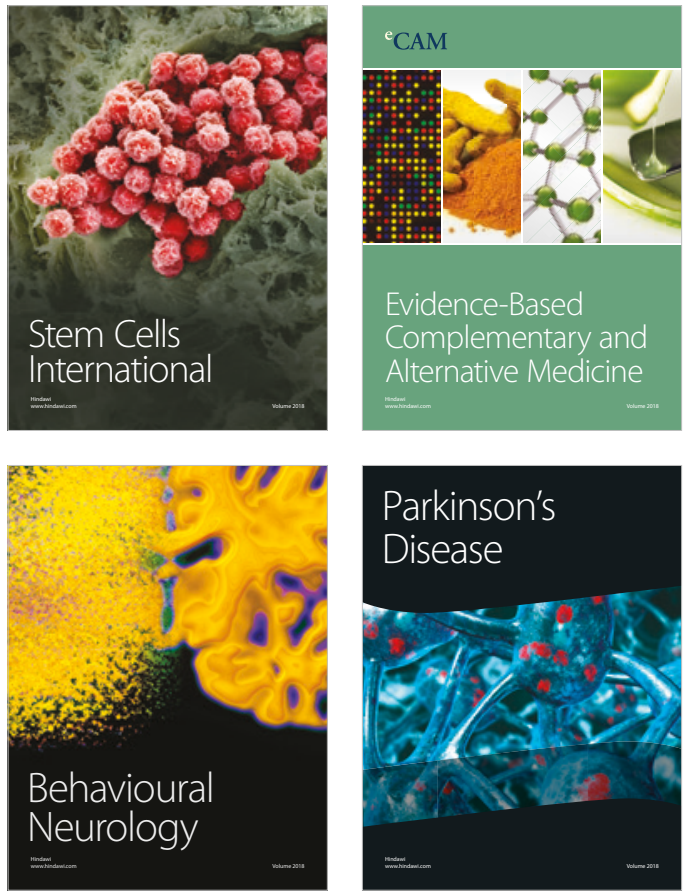

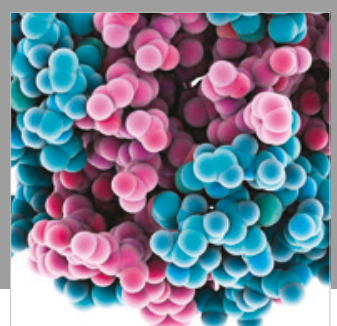

ournal of

Diabetes Research

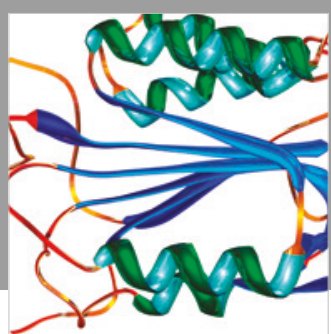

Disease Markers
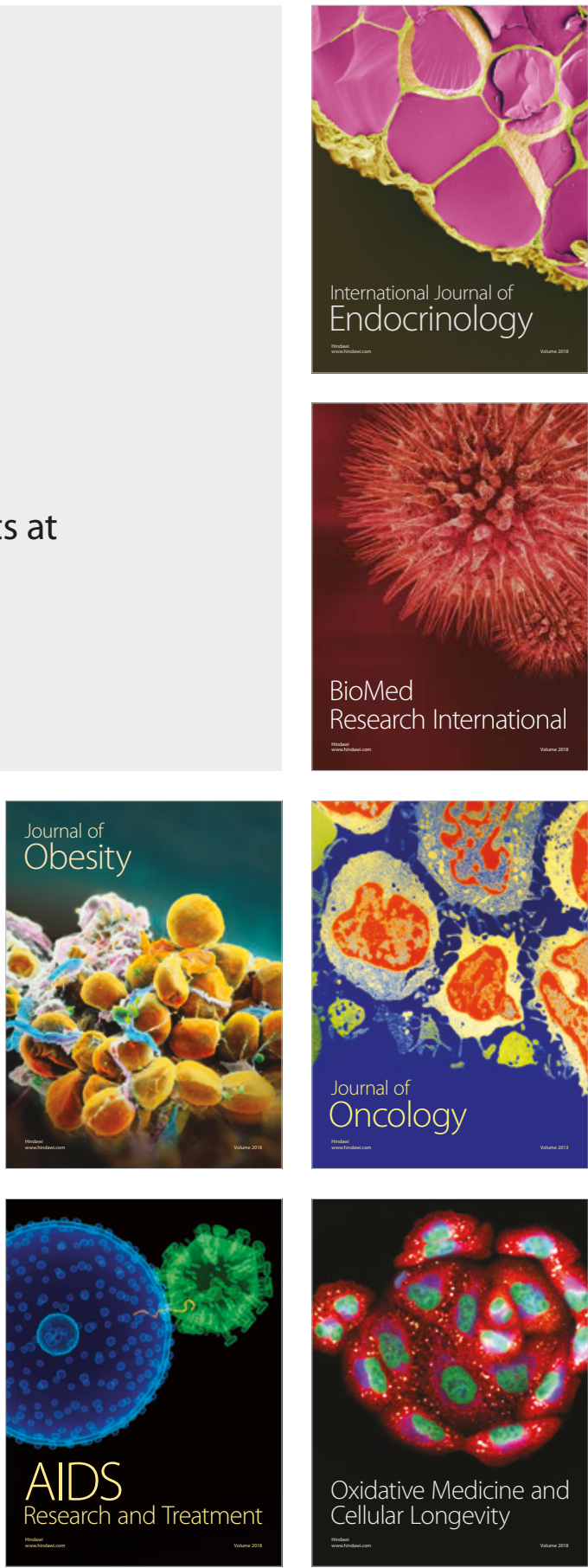